\title{
Immunotherapy in Patients with Lung Cancer with Driver Mutations: A Single-Centre Experience
}

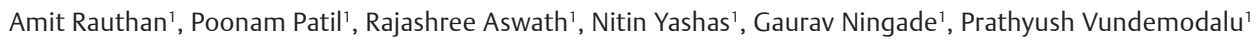

'Department of Medical Oncology, Manipal Hospitals, Bangalore, Karnataka, India

Address for correspondence: Prathyush Vundemodalu, MBBS, MD, Department of Medical Oncology, Manipal Hospitals, Bangalore, Karnataka, 560038, India (e-mail: prathyushv50@gmail.com)

\section{Abstract}

Introduction Immunotherapy has revolutionized treatment in metastatic nonsmall cell lung cancer (NSCLC) without driver mutations. Trial data shows that programmed death-1/PDL1 blockade in epidermal growth factor receptor (EGFR) and other driver mutation positive lung cancers is not beneficial; and instead maybe detrimental. Here, we evaluated the efficacy of immune check point inhibitors in a series of patients with EGFR and other driver mutation-positive advanced NSCLC.

Objectives This study was aimed to evaluate the efficacy of immune check point inhibitors in a series of patients with EGFR and other driver mutation-positive advanced NSCLC.

Materials and Methods We retrospectively analyzed 75 patients which received PD1/PDL1 inhibitors for advanced NSCL between January 2017 and January 2020. Ten patients were detected to have driver mutations on either tumor tissue or blood by next-generation sequencing (NGS). PDL1 status was assessed on SP263 ventana platform.

Results Out of 10 patients, 7 were male and 3 were female. EGFR was detected in six patients (three on tumor and three in blood NGS), MET exon 14 skipping mutation in two patients, and RAS mutation in two patients on NGS in blood. Immunotherapy combined with chemotherapy was given in 5 (50\%) patients, immunotherapy + bevacizumab + chemotherapy in two $(20 \%)$ and immunotherapy alone in three patients (30\%). Immunotherapy was started as first line in four patients as tumor tissue was negative for EGFR, ALK, and ROS1 by single gene testing. The remaining six patients received immunotherapy on progression in the second or subsequent lines. On NGS testing at progression, EGFR mutation was detected in one patient, MET exon 14 skip mutation was detected in two patients, and RAS mutation was detected in two patients. Immunotherapy alone was used in three patients in view of advanced age and multiple comorbidities. The median progression-free survival (PFS) was 5 months (range: $2-11$ months). Two patients who received chemotherapy + bevacizumab + immunotherapy continue to do well without progression at 9 months.

Conclusion PD1/PDL1 checkpoint inhibitors seem to have a limited impact in treatment in patients with driver mutations. Molecular testing by NGS is recommended either on tumor tissue or on blood by NGS if single gene testing for EGFR/ALK/ROS1 alterations is negative. We recommend not using single agent checkpoint inhibitors in molecular driven advanced NSCLC even with high PDL1 expression. We do see benefit in patients who received PD1/PDL1 inhibitors in combination with chemotherapy with bevacizumab. In conclusion, in patients with molecular-driven NSCLC who progress after standard therapy can be treated with PD1/PDL1 inhibitors, but this should always be given in combination with chemotherapy and bevacizumab.

Keywords: lung cancer, immunotherapy, EGFR mutation

\begin{tabular}{|c|c|c|c|c|c|c|c|c|}
\hline & \begin{tabular}{|l|} 
Age/ \\
sex
\end{tabular} & Driver mutation & $\begin{array}{l}\text { Mutation } \\
\text { detected }\end{array}$ & PDL 1 & $\begin{array}{l}\text { Immunotherapy given as } \\
\text { single vs. combination }\end{array}$ & $\begin{array}{l}\text { Line of } \\
\text { immunotherapy }\end{array}$ & $\begin{array}{l}\text { Initial } \\
\text { treatment }\end{array}$ & \begin{tabular}{|l} 
PFS on \\
immunotherapy
\end{tabular} \\
\hline 1 & $46 / \mathrm{M}$ & EGFR exon 19 del & $\begin{array}{l}\text { Tumor } \\
\text { tissue }\end{array}$ & $0 \%$ & $\begin{array}{l}\text { Atezolizumab + carboplatin + } \\
\text { paclitaxel + bevacizumab }\end{array}$ & Second & Osimertinib & $\begin{array}{l}\text { Not yet reached at } \\
9 \text { months follow-up }\end{array}$ \\
\hline 2 & $54 / \mathrm{F}$ & $\begin{array}{l}\text { EGFR I861p } \\
\text { (uncommon mutation) }\end{array}$ & $\begin{array}{l}\text { NGS on } \\
\text { blood }\end{array}$ & $0 \%$ & $\begin{array}{l}\text { Pembrolizumab + peme- } \\
\text { trexed + carboplatin }\end{array}$ & Second & $\begin{array}{l}\text { Chemotherapy } \\
\text { alone }\end{array}$ & 10 months \\
\hline 3 & $70 / \mathrm{F}$ & $\begin{array}{l}\text { EGFR I747p } \\
\text { (uncommon mutation) }\end{array}$ & $\begin{array}{l}\text { NGS on } \\
\text { tissue }\end{array}$ & $90 \%$ & $\begin{array}{l}\text { Pembrolizumab + peme- } \\
\text { trexed + carboplatin }\end{array}$ & First & & 8 months \\
\hline 4 & $70 / \mathrm{M}$ & EGFR fusion & $\begin{array}{l}\text { NGS on } \\
\text { blood }\end{array}$ & $50 \%$ & $\begin{array}{l}\text { Pembrolizumab + peme- } \\
\text { trexed + carboplatin }\end{array}$ & First & & 11 months \\
\hline 5 & $63 / \mathrm{M}$ & $\begin{array}{l}\text { MET exon } 14 \text { skip } \\
\text { mutation }\end{array}$ & $\begin{array}{l}\text { NGS on } \\
\text { blood }\end{array}$ & $0 \%$ & $\begin{array}{l}\text { Pembrolizumab + nabpacli- } \\
\text { taxel + carboplatin }\end{array}$ & First & & 5 months \\
\hline 6 & $58 / \mathrm{M}$ & $\begin{array}{l}\text { EGFR exon } 21 \\
\text { mutation }\end{array}$ & $\begin{array}{l}\text { Tumor } \\
\text { tissue }\end{array}$ & $0 \%$ & Nivolumab alone & Third & $\begin{array}{l}\text { Erlotinib, } \\
\text { followed by } \\
\text { chemotherapy }\end{array}$ & 4 months \\
\hline 7 & $66 / \mathrm{M}$ & NRAS G12D mutation & $\begin{array}{l}\text { NGS on } \\
\text { blood }\end{array}$ & $0 \%$ & $\begin{array}{l}\text { Atezolizumab + bevacizumab } \\
\text { + nabpaclitaxel + carboplatin }\end{array}$ & Second & $\begin{array}{l}\text { Pemetrexed + } \\
\text { carboplatin }\end{array}$ & $\begin{array}{l}\text { Not progressed at } \\
9 \text { months }\end{array}$ \\
\hline 8 & $68 / \mathrm{F}$ & RAS G12C mutation & $\begin{array}{l}\text { NGS on } \\
\text { blood }\end{array}$ & $60 \%$ & $\begin{array}{l}\text { Pembrolizumab + carbopla- } \\
\text { tin + pemetrexed }\end{array}$ & First & & 4 months \\
\hline 9 & $88 / \mathrm{M}$ & $\begin{array}{l}\text { MET exon } 14 \text { skip } \\
\text { mutation }\end{array}$ & $\begin{array}{l}\text { NGS on } \\
\text { tumor }\end{array}$ & $0 \%$ & Pembrolizumab alone & First & & 2 months \\
\hline 10 & $80 / \mathrm{M}$ & $\begin{array}{l}\text { EGFR exon } 20 \text { uncom- } \\
\text { mon mutation }\end{array}$ & $\begin{array}{l}\text { Tumor } \\
\text { tissue }\end{array}$ & $0 \%$ & Nivolumab alone & Fourth & $\begin{array}{l}\text { Chemotherapy } \\
\text { followed by } \\
\text { afatinib }\end{array}$ & 3 months \\
\hline
\end{tabular}

Abbreviations: EGFR, epidermal growth factor receptor; NGS, next-generation sequencing; NRAS, Neuroblastoma RAS viral oncogene homolog; RAS, rat sarcoma virus; PDL 1, programmed death-ligand 1; PFS, progression free survival. 